\title{
An Economic Analysis of Pervasive, Incentive-Based Demand Response
}

\author{
Tri Kurniawan Wijaya*, Matteo Vasirani*, Jonas Christoffer Villumsen ${ }^{\dagger}$, and Karl Aberer* \\ *School of Computer and Communication Sciences, EPFL, Switzerland \\ *IBM Research Ireland \\ Email: tri-kurniawan.wijaya@epfl.ch, matteo.vasirani@epfl.ch, jonasvil@ie.ibm.com,karl.aberer@epfl.ch
}

\begin{abstract}
Demand response (DR) emerges as one of the cheapest and greenest solutions to match supply and demand in the electricity sector. While DR has been focused on large and industrial consumers, pervasive implementation (by including residential consumers) is needed to maximize its potential. This paper presents theoretical analysis of pervasive, incentive-based DR from the economics perspective. Our analysis consider cases whether (1) DR is used to encourage consumers to decrease or increase their demand, and (2) utility companies have access to a single or multiple energy sources. We determine the necessary conditions and derive the optimal incentives to benefit from DR events.
\end{abstract}

\section{INTRODUCTION}

While there are two types of DR, i.e., price- and incentivebased [1]-[3], this paper focuses on incentive-based DR, where under flat-rate price, consumers are offered some incentives to alter their energy consumption. The incentives can be, for example, movie tickets, bill rebates, redeemable vouchers, or virtual currencies which can be converted into real products or other benefits (as in the concept of miles in airline marketing). In addition, messages sent to communicate upcoming DR events can also be seen as triggers as to when to act [4].

DR has long been focused on large, industrial consumers. To maximize its potential, however, DR implementation needs to be pervasive by attracting residential consumers as well. To this end, the vision of smart grids and deployment of smart meters provided a wide-open opportunity to include the participation of residential consumers. Consequently, deep insight about pervasive DR is necessary. For example, what are the necessary conditions to benefit from DR? How much incentives should be given to consumers?

However, these tasks are very challenging due to at least three reasons. First, utility companies acquire their energy supply from various sources. Some of them buy energy from the market, some own coal power plant, some use natural gas (or other sources), and some own several generators powered by different sources, which contribute differently to companies' profit. Second, DR can be used not only to induce lower energy consumption, but also to encourage higher consumption, especially in case of energy surplus in the grid. The surplus can happen, for example, when the sun shines (for area with high penetration of solar power) or when the wind blows strongly (for area with high penetration of wind power) but the demand is low. Third, there are a lot of uncertainties in consumer responses, since they are influenced by numerous factors, such as the incentives, time of day, day of the week, outdoor temperature, weather, holidays, or guests at home.

Overview of Contributions. This paper provides theoret- ical analysis of pervasive, incentive-based DR. Our analysis focuses more on the economic subsystem rather than the physical subsystem. ${ }^{1}$ We consider cases where DR is used as a mechanism to reduce and increase energy consumption. We determine the lower bound of the generation cost and consumer reduction (or increase) rate ${ }^{2}$ such that DR is still beneficial. Additionally, we derive the optimal incentives to maximize gains from DR events. We present our analysis in cases where utility companies have access to both, single and multiple energy sources. This paper provides a formalization and base model to analyze/understand DR incentive schemes further.

\section{KEY ASSUMPTIONS}

\section{A. Load Generation}

Let us assume that a utility company has access to a set of generators or energy sources $G$. For a particular time period, let:

- $\quad L_{g_{i}}$ be the load assigned to generator $g_{i} \in G$, and $L=\sum_{g_{i} \in G} L_{g_{i}}$

- $\quad \operatorname{cap}\left(g_{i}\right)$ be the capacity of generator $g_{i}$, and

- $\quad C_{g_{i}}\left(L^{\prime}\right)$ be the total cost of meeting load demand $L^{\prime}$ using generator $g_{i}$ for the discussed period.

To meet the load demand $L$, a utility company assigns $L$ to one or more generators ${ }^{3}$ depending on the capacity and generation cost. We assume that the utility company assigns (or, the market implicitly assigns) the load to the cheapest generator first, up to its capacity, before using the more expensive ones. Thus, for simplicity, we assume that the set of generators $G$ is ordered by its generation cost, i.e., $C_{g_{i}}\left(L^{\prime}\right) \leq C_{g_{j}}\left(L^{\prime}\right)$ for $i \leq j$. Thus, for $j=i+1$, we have $L_{g_{j}}>0$ iff $L_{g_{i}}=\operatorname{cap}\left(g_{i}\right)$ and $L>\sum_{1 \leq k \leq i} L_{g_{k}} \cdot{ }^{4}$ We also assume that there are no temporal constraints ${ }^{5}$ and

${ }^{1}$ While the physical subsystem considers hardware that physically produces and transmits electricity, the economic subsystem considers the actors that are involved in the production, trade, or consumption of electricity and their mutual relationships [5].

${ }^{2}$ It indicates how sensitive a consumer is to incentives (see also Section II-B). The higher the consumer reduction (or increase) rate, the more sensitive she is.

${ }^{3}$ We use the terms energy sources and generators interchangeably.

${ }^{4}$ Note that, we consider the ordering here only for the discussed time period. The ordering could change, of course, from one period to another depending on the generation cost.

${ }^{5}$ For example, generator ramp constraints and start-up constraints. Note that, although large power plants (coal/nuclear) have non-negligible ramping constraints or startup cost, small-scale distribution utility companies, who also serves consumers, typically use smaller generation units (e.g., gas-powered) with small startup costs and higher ramping capabilities. 
no appropriate energy storage solutions available to the utility company.

\section{B. Consumer Responses}

One of the main challenges faced by utility companies to carry out DR events for residential consumers is that only little is known about how consumers will respond to DR signals. Consumer responses can be affected by many factors, such as the amount of incentives, time of the day, day of the week, weather, outdoor temperature, holidays, or guests at home. Understanding the influence of these factors requires real implementation. However, utility companies need to have an understanding and holistic view about DR before starting any real implementation. Hence, we have a chicken and egg problem here.

Inspired by prior research on consumer response to dynamic pricing, which confirmed that consumer energy reduction increases as the electricity prices increases [6], we assume that consumer response grows proportionally with the amount of incentives, given other factors are held constant. For simplicity, below we specify consumer responses as demand reduction (see Section IV for consumer responses in the context of DR to increase demand). Let $\left\{x_{1}, x_{2}, \ldots, x_{n}\right\}$ be the set of factors that (possibly) influence consumer responses, and $x_{1}=\mathcal{I}$ be the incentives (unit: $\$$ ). ${ }^{6}$ We define consumer responses (or the demand reduction) during a DR event as:

$$
r\left(x_{1}, x_{2}, \ldots, x_{n}\right)=r\left(\mathcal{I}, x_{2}, \ldots, x_{n}\right)=m \mathcal{I}+c,
$$

where $m$ is the consumer reduction rate (unit: $k W h / \$$ ), and $c$ is a constant. We assume $m \geq 0$ and $\mathcal{I} \geq 0$. Note that, $m$ may vary depending on other factors $x_{2}, \ldots, x_{n}$ (e.g., time of day, day of week, temperature). Here, $m$ is the consumer reduction rate in the discussed period. ${ }^{7}$ We also assume that any reduction during a DR event requires some incentives, i.e., $c=0$. Thus, we have:

$$
r\left(\mathcal{I}, x_{2}, \ldots, x_{n}\right)=m \mathcal{I} .
$$

Intuitively, consumer reduction rate $m$ is the amount of demand reduction that can be obtained using a unit of incentive. It can also be thought of as $\frac{d r}{d \mathcal{I}}$, the rate of reduction per unit incentive. In the sequel, we often use $R$ instead of $r\left(\mathcal{I}, f_{1}, \ldots, f_{n}\right)$ to simplify notation.

\section{DR TO REDUCE ENERGY CONSUMPTION}

\section{A. Without DR (Business As Usual)}

Cost We define the cost to meet load demand $L$ as $\sum_{g_{i} \in G} C_{g_{i}}\left(L_{g_{i}}\right)$, where $L=\sum_{g_{i} \in G} L_{g_{i}}$.

Revenue Let $P_{\text {ret }}$ be the retail price (unit: $\$ / k W h$ ) paid by the consumers, and $L$ be the total demand. Thus, company revenue $=P_{\text {ret }} L$.

Profit We define company's profit as its revenue minus cost:

$$
P_{r e t} L-\sum_{g_{i} \in G} C_{g_{i}}\left(L_{g_{i}}\right)
$$

\footnotetext{
${ }^{6}$ In practice, the incentives can be of form movie tickets, bill rebates, redeemable vouchers, etc. For simplicity, we quantify $\mathcal{I}$ with its monetary value (unit: \$) - company's cost to provide the incentives.

${ }^{7}$ While identifying how $m$ varies depending on other factors is interesting by itself, but it is beyond the scope of this paper. In practice, it can be estimated by the demand reduction for a unit of incentive.
}

\section{B. With DR}

Let $\mathcal{I}$ be the incentives given to the consumers, and $R$ denotes the consumers' demand reduction in a particular DR event. Thus, the total demand in the presence of the event is $L^{D R}=L-R$.

Cost The cost of meeting the load demand $L^{D R}$ is

$\sum_{g_{i} \in G} C_{g_{i}}\left(L_{g_{i}}^{D R}\right)$, where $L^{D R}=\sum_{g_{i} \in G} L_{g_{i}}^{D R}$.

Revenue Company's revenue $=P_{\text {ret }} L^{D R}$.

Profit Company's profit $=$ revenue - cost - incentives:

$$
P_{r e t} L^{D R}-\sum_{g_{i} \in G} C_{g_{i}}\left(L_{g_{i}}^{D R}\right)-\mathcal{I}
$$

Gain We define utility company's gain from a DR event as the difference between its profit with and without the DR event, i.e., by substracting Eq. 3 from Eq. 4:

$$
\begin{aligned}
\text { gain }= & P_{\text {ret }} L^{D R}-\sum_{g_{i} \in G} C_{g_{i}}\left(L_{g_{i}}^{D R}\right)-\mathcal{I}-P_{r e t} L+ \\
& \sum_{g_{i} \in G} C_{g_{i}}\left(L_{g_{i}}\right) \\
= & \sum_{g_{i} \in G}\left(C_{g_{i}}\left(L_{g_{i}}\right)-C_{g_{i}}\left(L_{g_{i}}^{D R}\right)\right)- \\
& P_{\text {ret }}\left(L-L^{D R}\right)-\mathcal{I} \\
= & \sum_{g_{i} \in G}\left(C_{g_{i}}\left(L_{g_{i}}\right)-C_{g_{i}}\left(L_{g_{i}}^{D R}\right)\right)-P_{r e t} R-\mathcal{I} .
\end{aligned}
$$

If $L^{D R} \leq L$, then there are some generators that are unused or do not run at their full capacity. Let us denote this set of generators as $G_{r e d}$, i.e., the reduced generators. And, we define the rest of the generators as $G_{\text {base }}=G \backslash G_{\text {red }}$. Formally:

- $g_{i} \in G_{\text {red }}$, iff $L_{g_{i}}^{D R}<L_{g_{i}}$, and

- $g_{i} \in G_{\text {base }}$, iff $L_{g_{i}}^{D R}=L_{g_{i}}$.

For instance, when there is a demand reduction during a DR event, then by definition, the most expensive generator belongs to $G_{r e d}$. We can rewrite the gain computation in Eq. 5 by separating the set of generators in $G_{\text {base }}$ and $G_{\text {red }}$ as:

$$
\begin{aligned}
\text { gain }= & \sum_{g_{i} \in G}\left(C_{g_{i}}\left(L_{g_{i}}\right)-C_{g_{i}}\left(L_{g_{i}}^{D R}\right)\right)-P_{\text {ret }} R-\mathcal{I} \\
= & \sum_{g_{i} \in G_{\text {base }}}\left(C_{g_{i}}\left(L_{g_{i}}\right)-C_{g_{i}}\left(L_{g_{i}}^{D R}\right)\right)+ \\
& \sum_{g_{i} \in G_{\text {red }}}\left(C_{g_{i}}\left(L_{g_{i}}\right)-C_{g_{i}}\left(L_{g_{i}}^{D R}\right)\right)-P_{r e t} R-\mathcal{I} \\
= & \sum_{g_{i} \in G_{\text {red }}}\left(C_{g_{i}}\left(L_{g_{i}}\right)-C_{g_{i}}\left(L_{g_{i}}^{D R}\right)\right)-P_{\text {ret }} R-\mathcal{I}
\end{aligned}
$$

Thus, company's gain from a DR event depends on the (i) difference in the total cost of meeting the load demand, (ii) reduction in the revenue, and (iii) total incentives.

The next challenge is to determine just the right incentives to obtain positive gain (or benefit) from DR events. After identifying conditions to obtain positive gain, we aim to find the optimal incentives to maximize gain. To this end, we divide the problem into two cases, i.e., first, where a utility company has access to a single energy source, and second, in a more general setting, where a utility company has access to multiple energy sources.

\section{Single Energy Source}

In this section, we study more deeply the setting where a utility company has access to only one energy source, i.e., $|G|=1$. The energy source can be, for example, an energy market. Let $P_{m k t}$ be the unit cost to meet the load demand for the period under discussion, or the market price if the energy 
source is an energy market. Then, we can rewrite the gain computation in Eq. 6:

$$
\begin{aligned}
\text { gain } & =\sum_{g_{i} \in G_{\text {red }}}\left(C_{g_{i}}\left(L_{g_{i}}\right)-C_{g_{i}}\left(L_{g_{i}}^{D R}\right)\right)-P_{\text {ret }} R-\mathcal{I} \\
& =P_{m k t} L-P_{m k t} L^{D R}-P_{\text {ret }} R-\mathcal{I} \\
& =P_{m k t} R-P_{\text {ret }} R-\mathcal{I}=\left(P_{\text {mkt }}-P_{\text {ret }}\right) R-\mathcal{I} .
\end{aligned}
$$

Note that, when the energy source considered is indeed an energy market, then the market price before and after DR events might be different due to the changes in the demand. In this case, however, we assume that demand reduction $R$ does not influence the market price, i.e., it is much smaller compared to the overall demand in the market. Next, without loss of generality, we assume that the energy source is the market.

1) The lower bound of market price: By substituting $R$ from Eq. 2 into Eq. 7, we obtain:

$$
\begin{aligned}
\text { gain } & =\left(P_{\text {mkt }}-P_{\text {ret }}\right) m \mathcal{I}-\mathcal{I} \\
& =\left(\left(P_{\text {mkt }}-P_{\text {ret }}\right) m-1\right) \mathcal{I} .
\end{aligned}
$$

To have positive gain, $\left(P_{m k t}-P_{r e t}\right) m-1$ should be positive. Thus:

$$
P_{m k t}>\frac{1}{m}+P_{r e t} .
$$

Recall that the consumer reduction rate, $m$ (unit: $k W h / \$$ ), expresses the amount of energy a consumer willing to sacrifice for a unit of incentive during a DR event. In economics, $m$ is also known as consumer's willingness to pay, that is, the maximum amount of energy a consumer is willing to sacrifice from her normal consumption level for a unit incentive. On the other hand, $\frac{1}{m}$ (unit: $\$ / k W h$ ) is consumer willingness to accept, i.e., the minimum amount of incentive a consumer is willing to accept for a unit of energy she sacrifices from her normal consumption during a DR event.

Inequality in Eq. 9 states that DR can bring an advantage to utility companies when the market price is greater than the retail price plus the consumer willingness to accept. It provides deeper insight to the common belief that DR should be carried out when $P_{m k t}>P_{r e t}$. The customer willingness to accept should be taken into account as well, i.e., $P_{m k t}>\frac{1}{m}+P_{\text {ret }}$.

2) The lower bound of consumer reduction rate: From Eq. 9, we can also derive the lowest consumer reduction rate required to obtain positive gain:

$$
m>\frac{1}{P_{m k t}-P_{\text {ret }}} \text {. }
$$

That is, the higher the difference between the market and the retail price, the less sensitive the consumers that a company needs in its portfolio for a DR event, i.e., having consumers with low $m$ works just fine. However, when there is only a marginal difference between the market and the retail price, the company should prefer consumers that are highly sensitive to incentives.

3) The optimal incentives: When Eq. 9 or 10 is satisfied, setting larger $\mathcal{I}$ leads to higher gain (see Eq. 8). However, a larger $\mathcal{I}$ also causes a larger $R$. Thus, when $R>L$, it means that consumers give some energy back to the grid (or producing energy, i.e., becoming prosumers). Then, the (positive) gain is due to consumers' energy price, $\frac{1}{m}$, is cheaper than $P_{m k t}-P_{r e t}$. However, demand reduction is typically limited. This can be, for example, because the grids accept only limited bidirectional energy flow, or consumers (distributed) energy generation capacity are limited. Let $R_{\max }$ denotes the consumers' maximum reduction, where $R \leq R_{\max }$ :

$$
R= \begin{cases}m \mathcal{I}, & \text { if } \mathcal{I} \leq \frac{R_{\max }}{m}, \\ R_{\max }, & \text { if } \mathcal{I}>\frac{R_{\max }}{m} .\end{cases}
$$

In most cases, it is easier to estimate the base load $L_{\min }$, the minimum amount of electricity the consumers cannot live without. Thus, one can estimate $R_{\max }$ by computing $R_{\max }=$ $L-L_{\min }$.

Theorem 1: In case of single energy source, when consumer reduction is bounded by $R_{\max }$ and Eq. 9 or 10 is satisfied, then the incentives that maximize company's gain is:

$$
\mathcal{I}_{\text {opt }}=\frac{R_{\max }}{m}
$$

Proof: Let gain $(\star)$ be the company's gain using incentives $\star$. Then, there are two cases:

Case 1: The company gives incentives $\mathcal{I}^{\prime}<\mathcal{I}_{\text {opt }}$. Let $R^{\prime}=m \mathcal{I}^{\prime}$. Below, we show that $\operatorname{gain}\left(\mathcal{I}^{\prime}\right)<\operatorname{gain}\left(\mathcal{I}_{\text {opt }}\right)$.

$$
\begin{aligned}
\operatorname{gain}\left(\mathcal{I}^{\prime}\right) & =\left(P_{m k t}-P_{\text {ret }}\right) R^{\prime}-\mathcal{I}^{\prime} \\
& =\left(P_{m k t}-P_{r e t}\right) m \mathcal{I}^{\prime}-\mathcal{I}^{\prime} \\
& <\left(\left(P_{m k t}-P_{r e t}\right) m \mathcal{I}^{\prime}-\mathcal{I}^{\prime}\right) \frac{\mathcal{I}_{\text {opt }}}{\mathcal{I}^{\prime}} \\
& =\left(P_{m k t}-P_{r e t}\right) m \mathcal{I}_{o p t}-\mathcal{I}_{o p t} \\
& =\operatorname{gain}\left(\mathcal{I}_{o p t}\right) .
\end{aligned}
$$

Case 2: The company gives incentives $\mathcal{I}^{\prime}>\mathcal{I}_{\text {opt }}$. Since $\mathcal{I}^{\prime}>\frac{R_{\max }}{m}$, then according to Eq. 11 , the demand reduction $R^{\prime}=R_{\max }^{m}$. Below, we show that $\operatorname{gain}\left(\mathcal{I}^{\prime}\right)<\operatorname{gain}\left(\mathcal{I}_{\text {opt }}\right)$.

$$
\begin{aligned}
\operatorname{gain}\left(\mathcal{I}^{\prime}\right) & =\left(P_{m k t}-P_{\text {ret }}\right) R^{\prime}-\mathcal{I}^{\prime} \\
& =\left(P_{m k t}-P_{\text {ret }}\right) R_{\text {max }}-\mathcal{I}^{\prime} \\
& <\left(P_{m k t}-P_{\text {ret }}\right) R_{\text {max }}-\mathcal{I}_{\text {opt }} \\
& =\left(P_{m k t}-P_{\text {ret }}\right) m \mathcal{I}_{\text {opt }}-\mathcal{I}_{\text {opt }} \\
& =\operatorname{gain}\left(\mathcal{I}_{\text {opt }}\right) .
\end{aligned}
$$

4) The upper bound of incentives: To obtain the highest incentive which still gives us positive gain, we require the gain to be greater than 0 while considering the maximum reduction $R_{\max }$. We replace $R$ in Eq. 7 with $R_{\max }$ :

$$
\begin{aligned}
\left(P_{\text {mkt }}-P_{\text {ret }}\right) R_{\max }-\mathcal{I} & >0 \\
\left(P_{\text {mkt }}-P_{\text {ret }}\right) R_{\max } & >\mathcal{I} .
\end{aligned}
$$

Example 1: While typically the market price is lower than the retail price, we consider in this example a period where the market price is higher than the retail price. Let the market price $P_{m k t}=1 \$ / k W h$, the retail price $P_{r e t}=0.2 \$ / k W h$, and $m=$ $2 k W h / \$$. Thus, inequalities in Eq. 9 and 10 are met. Suppose that the total load demand is $1000 \mathrm{kWh}$ and $L_{\min }=200 \mathrm{kWh}$. Then, we have $R_{\max }=1000-200=800 \mathrm{kWh}$. Figure 1 illustrates company's gain for different amount of incentives given to customers. Maximum gain is obtained where the incentive is equal to $\mathcal{I}_{\text {opt }}=R_{\max } / m=400 \$$. And, the upper bound of the incentives such that the company still experiences positive gain is $\left(P_{m k t}-P_{r e t}\right) R_{\max }=640 \$$. 


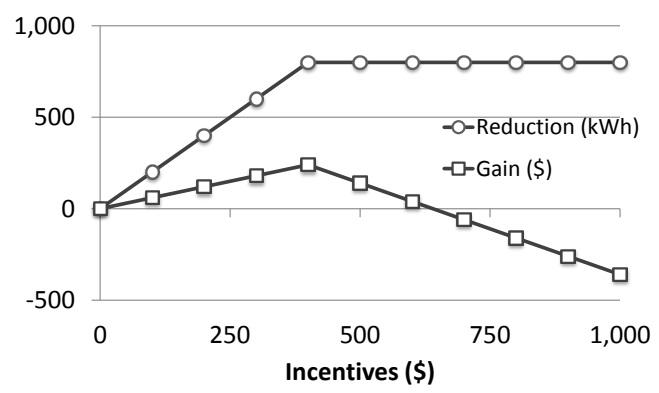

Fig. 1: Company's gain for different incentives, where $P_{\text {ret }}=$ $0.2 \$ / k W h, P_{m k t}=1 \$ / k W h, m=2 k W h / \$$, and $R_{m a x}=800 k W h$. The optimal incentive is $400 \$$, while the highest incentive such that the company still experiences positive gain is $640 \$$.

\section{Multiple Energy Sources}

In this section, we discuss the case of utility companies who have access to multiple energy sources. ${ }^{8}$ Let us define $R_{g_{i}}$ as the demand reduction for generator $g_{i}$, that is, $R_{g_{i}}=L_{g_{i}}-$ $L_{g_{i}}^{D R}$, and $\mathcal{I}_{g_{i}}=R_{g_{i}} / m$. Recall that, using the definition of $G_{\text {red }}$ and $G_{\text {base }}$, we can rewrite the demand reduction as $R=$ $\sum_{g_{i} \in G} R_{g_{i}}=\sum_{g_{i} \in G_{r e d}} R_{g_{i}}$. Similarly, we can also rewrite the incentives as:

$$
\begin{aligned}
R & =\sum_{g_{i} \in G_{\text {red }}} R_{g_{i}} \\
m \mathcal{I} & =\sum_{g_{i} \in G_{\text {red }}}\left(m \mathcal{I}_{g_{i}}\right) \\
\mathcal{I} & =\sum_{g_{i} \in G_{\text {red }}} \mathcal{I}_{g_{i}} .
\end{aligned}
$$

Suppose that the unit cost to meet load demand for the discussed period using generator $g_{i}$ is $P_{g_{i}}$, or $C_{g_{i}}\left(L_{g_{i}}\right)=P_{g_{i}} L_{g_{i}}$. Note that, this assumption makes the cost function linear. However, sometimes we also would like to express the cost function as a step function. In this case, we could formulate the step function by considering each step as a distinct generator. Some studies also consider quadratic cost function [7]-[9]. Since a quadratic function can be approximated by a step function, our formulation above also allows us to approximate quadratic cost functions as well. We could then rewrite the gain computation in Eq. 6 as:

$$
\begin{aligned}
\text { gain } & =\sum_{g_{i} \in G_{\text {red }}}\left(P_{g_{i}} L_{g_{i}}-P_{g_{i}} L_{g_{i}}^{D R}\right)-P_{\text {ret }} R-\mathcal{I} \\
& =\sum_{g_{i} \in G_{\text {red }}}\left(P_{g_{i}} R_{g_{i}}\right)-P_{\text {ret }} R-\mathcal{I} \\
& =\sum_{g_{i} \in G_{\text {red }}}\left(P_{g_{i}} R_{g_{i}}\right)-\sum_{g_{i} \in G_{\text {red }}}\left(P_{\text {ret }} R_{g_{i}}\right)-\sum_{g_{i} \in G_{\text {red }}} \mathcal{I}_{g_{i}} \\
& =\sum_{g_{i} \in G_{\text {red }}}\left(\left(P_{g_{i}}-P_{\text {ret }}\right) R_{g_{i}}-\mathcal{I}_{g_{i}}\right)
\end{aligned}
$$

Further, we denote gain $_{g_{i}}=\left(P_{g_{i}}-P_{\text {ret }}\right) R_{g_{i}}-\mathcal{I}_{g_{i}}$ as the gain contributed by generator $g_{i}$.

1) Meaningful DR events: We define meaningful demand reduction as the reduction that yields positive gain, whereas demand reduction that yields zero or negative gain is meaningless. Additionally, a DR event is meaningful if and only if the demand reduction of each generator is meaningful. ${ }^{9}$ Thus, it implies that any effort and incentive given to customers should

\footnotetext{
${ }^{8}$ Note that, an energy market could also be one of them.

${ }^{9}$ It is also possible to define meaningful DR events in a weaker sense, i.e., if only if the event yield a positive gain. If we define it that way, however, it means that there could be a set of generators $G_{\text {red }}^{+}$where gain $_{g_{i}}>0$ for all $g_{i} \in G_{\text {red }}^{+}$and a set of generators $G_{\text {red }}^{-}$where gain $_{g_{i}} \leq 0$ for all $g_{i} \in G_{\text {red }}^{-}$. Since our goal is maximizing the gain, there is no point in considering (to reduce the load of) $G_{r e d}^{-}$. This is the reason we define meaningful DR events in its stronger sense, considering only $G_{r e d}^{+}$.
}

contribute to company's gain:

$$
\begin{aligned}
\forall g_{i} \in G_{\text {red }}, & \text { gain }_{g_{i}}>0, \\
& \left(P_{g_{i}}-P_{\text {ret }}\right) R_{g_{i}}-\mathcal{I}_{g_{i}}>0, \\
& \left(P_{g_{i}}-P_{\text {ret }}\right) m \mathcal{I}_{g_{i}}-\mathcal{I}_{g_{i}}>0, \\
& \left(\left(P_{g_{i}}-P_{r e t}\right) m-1\right) \mathcal{I}_{g_{i}}>0 .
\end{aligned}
$$

To have positive gain, $\left(\left(P_{g_{i}}-P_{r e t}\right) m-1\right)$ should be positive. Thus:

$$
\forall g_{i} \in G_{\text {red }}, \quad P_{g_{i}}>\frac{1}{m}+P_{r e t}, \text { or } m>\frac{1}{P_{g_{i}}-P_{r e t}} .
$$

2) The optimal incentives: Providing that the inequalities in Eq. 16 are satisfied, we can derive the optimal incentive.

Lemma 2: In case of a utility company with multiple energy sources, if demand reduction $R$ is unbounded and Eq. 16 is satisfied, then higher incentives $\mathcal{I}$ leads to higher demand reduction $R$, and eventually higher gain.

Proof: Let gain $(\mathcal{I})$ be the gain using incentives $\mathcal{I}$ and gain $_{g_{i}}\left(\mathcal{I}_{g_{i}}\right)$ be the gain contributed by generator $g_{i}$ using incentives $\mathcal{I}_{g_{i}}$. Therefore, gain $(\mathcal{I})=\sum_{g_{i} \in G_{\text {red }}}$ gain $_{g_{i}}\left(\mathcal{I}_{g_{i}}\right)$ (see also Eq. 14).

We need to show that whenever $\mathcal{I}>\mathcal{I}^{\prime}$, then $\operatorname{gain}(\mathcal{I})>$ $\operatorname{gain}\left(\mathcal{I}^{\prime}\right)$. We assume that whether the company gives $\mathcal{I}$ or $\mathcal{I}^{\prime}$, Eq. 16 is satisfied. Let $R=m \mathcal{I}$ and $R^{\prime}=m \mathcal{I}^{\prime}$ be the demand reduction and $G_{\text {red }}$ and $G_{\text {red }}^{\prime}$ be the set of reduced generators when the utility company gives incentives $\mathcal{I}$ and $\mathcal{I}^{\prime}$, respectively. Because $R>R^{\prime}$, then $G_{r e d} \supseteq G_{r e d}^{\prime}$. Then, there are two cases:

Case 1: $G_{\text {red }}=G_{\text {red }}^{\prime}$. This means that we have the same amount of reduction in every generators, in $G_{\text {red }}$ and $G_{\text {red }}^{\prime}$, up to their capacity, except for the cheapest one (since $R>R^{\prime}$ ). Let $g_{\omega}$ be the cheapest generator in $G_{r e d}$ (and in $G_{\text {red }}^{\prime}$ ). Then, $\forall g_{i} \in G_{r e d} \backslash g_{\omega}$, we have $R_{g_{i}}=R_{g_{i}}^{\prime}=\operatorname{cap}\left(g_{i}\right)$. Consequently, since $R>R^{\prime}$, we have $R_{g_{\omega}}>R_{g_{\omega}}^{\prime}$. And since $\mathcal{I}_{g_{i}}=R_{g_{i}} / m$ and $\mathcal{I}_{g_{i}}^{\prime}=R_{g_{i}}^{\prime} / m$, we have $\mathcal{I}_{g_{\omega}}>\mathcal{I}_{g_{\omega}}^{\prime}$ and gain $_{g_{\omega}}\left(\mathcal{I}_{g_{\omega}}\right)>\operatorname{gain}_{g_{\omega}}\left(\mathcal{I}_{g_{\omega}}^{\prime}\right)$. Thus:

$$
\begin{aligned}
\operatorname{gain}(\mathcal{I}) & =\sum_{g_{i} \in G_{r e d} \backslash g_{\omega}} \text { gain }_{g_{i}}\left(\mathcal{I}_{g_{i}}\right)+\text { gain }_{g_{\omega}}\left(\mathcal{I}_{g_{\omega}}\right) \\
& >\sum_{g_{i} \in G_{r e d} \backslash g_{\omega}} \operatorname{gain}_{g_{i}}\left(\mathcal{I}_{g_{i}}\right)+\operatorname{gain}_{g_{\omega}}\left(\mathcal{I}_{g_{\omega}}^{\prime}\right) \\
& =\sum_{g_{i} \in G_{\text {red }}^{\prime} \backslash g_{\omega}} \text { gain }_{g_{i}}\left(\mathcal{I}_{g_{i}}^{\prime}\right)+\operatorname{gain}_{g_{\omega}}\left(\mathcal{I}_{g_{\omega}}^{\prime}\right) \\
& =\operatorname{gain}_{\left(\mathcal{I}^{\prime}\right)}
\end{aligned}
$$

Case 2: $G_{\text {red }} \supset G_{\text {red }}^{\prime}$. Since the inequalities in Eq. 16 is satisfied, $\forall g_{i} \in G_{\text {red }} \backslash G_{\text {red }}^{\prime}$, we have gain $_{g_{i}}\left(\mathcal{I}_{g_{i}}\right)>0$. Thus:

$$
\begin{aligned}
\operatorname{gain}(\mathcal{I}) & =\sum_{g_{i} \in G_{\text {red }}} \operatorname{gain}_{g_{i}}\left(\mathcal{I}_{g_{i}}\right) \\
& =\sum_{g_{i} \in G_{\text {red }}^{\prime}} \text { gain }_{g_{i}}\left(\mathcal{I}_{g_{i}}\right)+\sum_{g_{i} \in G_{\text {red }} \backslash G_{\text {red }}^{\prime}} \operatorname{gain}_{g_{i}}\left(\mathcal{I}_{g_{i}}\right) \\
& >\sum_{g_{i} \in G_{\text {red }}^{\prime}} \operatorname{gain}_{g_{i}}\left(\mathcal{I}_{g_{i}}\right) \\
& =\operatorname{gain}\left(\mathcal{I}^{\prime}\right)
\end{aligned}
$$

Similar to the case of single energy source, however, consumer demand reduction is typically not unbounded. Let $R_{\max }$ denotes the upper bound of the reduction (see also Eq. 11).

Theorem 3: When demand reduction is bounded by $R_{\max }$ and Eq. 16 is satisfied, then the optimal incentives that maximize company's gain is: $\mathcal{I}_{\text {opt }}=R_{\max } / \mathrm{m}$. 
Proof: Let gain $(\mathcal{I})$ denotes the gain using incentives $\mathcal{I}$. We show that when a utility company gives incentives $\mathcal{I}^{\prime} \neq$ $\mathcal{I}_{\text {opt }}$, then $\operatorname{gain}\left(\mathcal{I}^{\prime}\right)<\operatorname{gain}\left(\mathcal{I}_{\text {opt }}\right)$. Let $R^{\prime}=m \mathcal{I}^{\prime}$ and $R_{\text {opt }}=$ $m \mathcal{I}_{\text {opt }}$ be the demand reduction and $G_{\text {red }}^{\prime}$ and $G_{\text {red }}$ be the set of reduced generators when the company gives incentives $\mathcal{I}^{\prime}$ and $\mathcal{I}_{\text {opt }}$, respectively. Then, there are two cases:

Case 1: The company gives incentives $\mathcal{I}_{\text {opt }}>\mathcal{I}^{\prime}$. We need to show that $\operatorname{gain}\left(\mathcal{I}_{\text {opt }}\right)>\operatorname{gain}\left(\mathcal{I}^{\prime}\right)$. Note that, in this case, we have $R>R^{\prime}$, which also implies $G_{\text {red }} \supseteq G_{\text {red }}^{\prime}$. Then, we proceed as in the proof of Lemma 2, Case 1 and Case 2, by substituting $\mathcal{I}$ with $\mathcal{I}_{o p t}$ and $R$ with $R_{o p t}$.

Case 2: The company gives incentives $\mathcal{I}_{\text {opt }}<\mathcal{I}^{\prime}$. We need show that $\operatorname{gain}\left(\mathcal{I}_{\text {opt }}\right)>\operatorname{gain}\left(\mathcal{I}^{\prime}\right)$. Since $\mathcal{I}^{\prime}>\mathcal{I}_{\text {opt }}=$ $R_{\max } / m$, in case of bounded reduction (see Eq. 11), we have $R^{\prime}=R_{\text {max }}=m \mathcal{I}_{\text {opt }}=R_{\text {opt }}$. This also implies that $G_{r e d}^{\prime}=$ $G_{\text {red }}$.

$$
\begin{aligned}
\operatorname{gain}\left(\mathcal{I}^{\prime}\right) & =\sum_{g_{i} \in G_{\text {red }}^{\prime}}\left(P_{g_{i}}-P_{\text {ret }}\right) R^{\prime}-\mathcal{I}^{\prime} \\
& <\sum_{g_{i} \in G_{\text {red }}^{\prime}}\left(P_{g_{i}}-P_{\text {ret }}\right) R^{\prime}-\mathcal{I}_{\text {opt }} \\
& =\sum_{g_{i} \in G_{\text {red }}}\left(P_{g_{i}}-P_{\text {ret }}\right) R_{\text {opt }}-\mathcal{I}_{\text {opt }} \\
& =\operatorname{gain}\left(\mathcal{I}_{\text {opt }}\right) .
\end{aligned}
$$

3) The upper bound of incentives: When the demand reduction is maximum, i.e., $R_{\max }=L-L^{D R}$, we denote $R_{g_{i}}^{\max }=L_{g_{i}}-L_{g_{i}}^{D R}$ as the demand reduction for generator $g_{i}$. Consequently, we have $R_{\max }=\sum_{g_{i} \in G_{\text {red }}} R_{g_{i}}^{\max }$. Then, by replacing $R_{g_{i}}$ in Eq. 14 with $R_{g_{i}}^{\max }$, we can derive the highest incentives a company could provide while maintaining positive gain:

$$
\begin{aligned}
\sum_{g_{i} \in G_{\text {red }}}\left[\left(P_{g_{i}}-P_{\text {ret }}\right) R_{g_{i}}^{\max }-\mathcal{I}_{g_{i}}\right] & >0 \\
\sum_{g_{i} \in G_{\text {red }}}\left[\left(P_{g_{i}}-P_{r e t}\right) R_{g_{i}}^{\text {max }}\right]-\mathcal{I} & >0 \\
\sum_{g_{i} \in G_{\text {red }}}\left(P_{g_{i}}-P_{\text {ret }}\right) R_{g_{i}}^{\text {max }} & >\mathcal{I} .
\end{aligned}
$$

Example 2: Suppose that a utility company has access to two different energy sources, $g_{1}$ and $g_{2}$, where $\operatorname{cap}\left(g_{1}\right)=$ $5000 \mathrm{~kW}$ and $\operatorname{cap}\left(g_{2}\right)=2000 \mathrm{~kW}$. Assume that for a specific time period, we have load demand $L=6500 \mathrm{kWh}$, $P_{g_{1}}=0.1 \$ / k W h$ (base generator), and $P_{g_{2}}=1 \$ / k W h$ (peak generator). Additionally, let $P_{\text {ret }}=0.2 \$ / k W h, m=2 k W h / \$$, and $R_{\max }=1200 \mathrm{kWh}$. Figure 2 shows company's gain for different amount of incentives given to the customers. Maximum gain is obtained when the incentive is equal to $\mathcal{I}_{\text {opt }}=R_{\text {max }} / m=600 \$$. The highest incentive such that the company still experiences positive gain is $\left(P_{g_{2}}-P_{r e t}\right) R_{g_{2}}^{\max }=$ $(1-0.2) \cdot 1200=960 \$ .{ }^{10}$

\section{DR TO INCREASE ENERGY CONSUMPTION}

In the previous section, we discuss about DR for energy reduction. However, DR could also be used to incentivize consumers to increase their energy consumption, especially when there is a surplus energy and balancing the surplus is costly. This can be, for example, when (the output of) some generators cannot be turned off (or reduced) easily without additional cost. Or, when the demand is surprisingly low, much less than the amount of energy bought in the day-ahead

\footnotetext{
${ }^{10}$ Note that, to compute $R_{g_{2}}^{\max }$, we need to first compute $L^{D R}$ as if the reduction is $R_{\max }$. Thus, $L^{\mathrm{DR}}=L-R_{\max }=5300 \mathrm{kWh}$. Using the load generation assumption in Section II-A, we have $L_{g_{1}}=5000 \mathrm{kWh}$, $L_{g_{2}}=1500 \mathrm{kWh}, L_{g_{1}}^{D R}=5000 \mathrm{kWh}$, and $L_{g_{2}}^{D R}=300 \mathrm{kWh}$. Thus, $R_{g_{1}}^{\max }=0 \mathrm{kWh}$ and $R_{g_{2}}^{g_{1}}=1200 \mathrm{kWh}$.
}

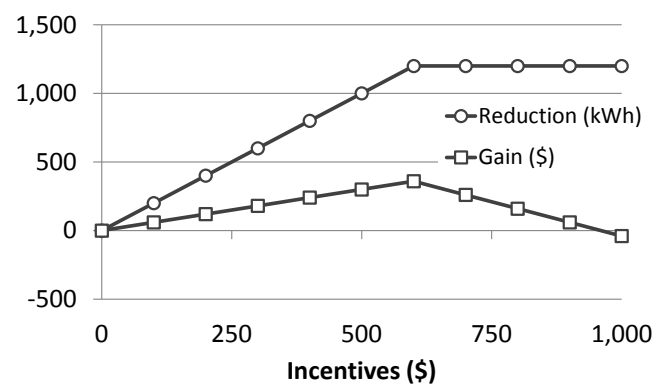

Fig. 2: Company's gain for different incentives where the company has two energy sources, $g_{1}$ and $g_{2}$, with $\operatorname{cap}\left(g_{1}\right)=500 \mathrm{~kW}$, $\operatorname{cap}\left(g_{2}\right)=5000 \mathrm{~kW}$. For this time period, we have $L=2000 \mathrm{kWh}$, $P_{g_{1}}=0.1 \$ / k W h, P_{g_{2}}=1 \$ / k W h, P_{r e t}=0.2 \$ / k W h, m=$ $2 \mathrm{kWh} / \$$, and $R_{\max }=1200 \mathrm{kWh}$. The optimal incentive is $600 \$$, while the highest incentive such that company still experiences positive gain is $960 \$$.

market. If the grid operator has to balance the surplus, then the company needs to pay the penalty for the imbalances.

Together with its ability to reduce demand, DR's ability to increase it forms a framework for a load shaping strategy. In this section, we discuss the case where DR is used as a mechanism to encourage demand increase. We present only the case where utility companies have access to multiple energy sources, since the case of single energy source can be regarded as a special case of that.

Let $L^{+}$be the total energy produced in the surplus/abundance period, where $L^{+}>L$, and $S_{\max }=L^{+}-L$ be the excess energy. Additionally, let $L_{g_{i}}^{+}$be the energy produced by generator $g_{i}$. We define $C_{B}\left(S_{\max }\right)$ as the balancing cost, e.g., the cost to balance the grid due to the excess energy (it is generally performed in a balancing market). Note that, in Section III, the role of the balancing market to generate additional electricity when the supply is short can also be seen as one of (typically more expensive) energy sources.

\section{A. Without DR (Business As Usual)}

Cost We define company's total cost as the sum of generation and balancing cost: $\sum_{g_{i} \in G} C_{g_{i}}\left(L_{g_{i}}^{+}\right)+C_{B}\left(S_{\max }\right)$.

Revenue Company's revenue: $P_{\text {ret }} L$.

Profit Company's profit $=$ revenue - cost:

$$
P_{r e t} L-\sum_{g_{i} \in G} C_{g_{i}}\left(L_{g_{i}}^{+}\right)-C_{B}\left(S_{\max }\right) .
$$

\section{B. With DR}

Note that, in this case there is a surplus of energy, $S_{\max }$, that is ready to be consumed. Encouraging customers to consume this surplus energy not only results in increasing company's revenue (due to higher customers' energy bill and lower balancing cost), but also acts as an energy balancing mechanisms, if there would be a shortage of energy in the future. It might be easier for people to consume less energy in the future (when the supply is short), if some activities have been performed or shifted to the previous (energy abundance) period. Therefore, we aim to use DR for incentivizing consumers to increase their consumption during this surplus period. Let us assume that the consumers increase their demand from $L$ to $L+S$, where $0 \leq S \leq S_{\max }$. 
Cost Compared to the previous case (without DR), company's cost is lower due to the possible decrease in the balancing cost: $\sum_{g_{i} \in G} C_{g_{i}}\left(L_{g_{i}}^{+}\right)+C_{B}\left(S_{\max }-S\right)$.

Revenue Company's revenue increases due to the possible increase in consumers' demand: $P_{\text {ret }}(L+S)$.

Profit Company's profit $=$ revenue - cost - incentive:

$$
P_{\text {ret }}(L+S)-\sum_{g_{i} \in G} C_{g_{i}}\left(L_{g_{i}}^{+}\right)-C_{B}\left(S_{\max }-S\right)-\mathcal{I} .
$$

Gain We define the gain of a utility company during a DR event in this abundance period as the difference between its profit with and without the DR event, i.e., Eq. 19 - Eq 18:

$$
\begin{aligned}
\text { gain }= & P_{\text {ret }}(L+S)-\sum_{g_{i} \in G} C_{g_{i}}\left(L_{g_{i}}^{+}\right)-C_{B}\left(S_{\max }-S\right)- \\
& \mathcal{I}-P_{\text {ret }} L+\sum_{g_{i} \in G} C_{g_{i}}\left(L_{g_{i}}^{+}\right)+C_{B}\left(S_{\max }\right) \\
= & P_{\text {ret }} S-\mathcal{I}-C_{B}\left(S_{\max }-S\right)+C_{B}\left(S_{\max }\right)
\end{aligned}
$$

As in the case of the generation cost (see also Section III-D), let us assume that the balancing cost has a unit cost $P_{B}$. Then, we have:

$$
\begin{aligned}
\text { gain } & =P_{\text {ret }} S-\mathcal{I}-P_{B}\left(S_{\max }-S\right)+P_{B} \cdot S_{\max } \\
& =P_{\text {ret }} S-\mathcal{I}+P_{B} S \\
& =\left(P_{\text {ret }}+P_{B}\right) S-\mathcal{I}
\end{aligned}
$$

Similar to the case of DR to reduce energy consumption, we assume that consumer responses grow linearly with the incentives offered, i.e., $S=m^{+} \cdot \mathcal{I}$, where $m^{+}$is the consumer increase rate (unit: $k W h / \$$ ). The higher the consumer increase rate, the more sensitive she is to incentives. ${ }^{11}$ Since $S$ is bounded by $S_{\max }$, consequently,

$$
S= \begin{cases}m^{+} \cdot \mathcal{I}, & \text { if } \mathcal{I} \leq \frac{S_{\max }}{m^{+}} \\ S_{\max }, & \text { if } \mathcal{I}>\frac{S_{\max }}{m^{+}} .\end{cases}
$$

1) The lower bound of consumer increase rate: In order to derive the lowest consumer increase rate required to obtain positive gain:

$$
\begin{aligned}
\left(P_{\text {ret }}+P_{B}\right) S-\mathcal{I} & >0 \\
\left(P_{\text {ret }}+P_{B}\right) m^{+} \cdot \mathcal{I}-\mathcal{I} & >0 \\
\left(\left(P_{\text {ret }}+P_{B}\right) m^{+}-1\right) \mathcal{I} & >0
\end{aligned}
$$

To have positive gain, the term $\left(P_{\text {ret }}+P_{B}\right) m^{+}-1$ should be positive. Thus:

$$
m^{+}>\frac{1}{P_{r e t}+P_{B}}
$$

If we assume that the retail price $P_{r e t}$ is fixed, then the sensitivity of the consumers required for a DR event is inversely related with the balancing prices. While highly sensitive consumers are generally preferred for DR, having less sensitive consumers (with low $\mathrm{m}^{+}$) is also fine when the balancing price is high enough.

\footnotetext{
${ }^{11}$ In practice, consumers can increase their demand by shifting their later activities to the DR event period. If they do not have any activities to be shifted, however, rational consumers would have $1 / m^{+}>P_{\text {ret }}$, i.e., they respond (or increase their demand) iff the incentive per unit energy is greater than the retail price.
}

2) The lower bound of the balancing price: Using Eq. 24, we can also derive the lower bound of the balancing price:

$$
P_{B}>\frac{1}{m^{+}}-P_{\text {ret }}
$$

3) The optimal incentives: When Eq. 24 or 25 is satisfied, we can derive the optimal incentives that maximize company's gain.

Theorem 4: In the case of DR to increase energy consumption, given that Eq. 24 or 25 is satisfied, the optimal incentives that maximize company's gain is: $\mathcal{I}_{o p t}=S_{\max } / m^{+}$.

Proof: Let $\operatorname{gain}(\mathcal{I})$ denotes company's gain by providing incentives $\mathcal{I}$. If Eq. 24 or 25 is satisfied, then for $\mathcal{I}^{\prime} \neq \mathcal{I}_{\text {opt }}$, we show that $\operatorname{gain}\left(\mathcal{I}^{\prime}\right)<\operatorname{gain}\left(\mathcal{I}_{\text {opt }}\right)$. Let $S^{\prime}=m^{+} \cdot \mathcal{I}^{\prime}$ be the increase in energy consumption due to incentives $\mathcal{I}^{\prime}$. Then, there are two cases:

$$
\begin{aligned}
\operatorname{Case} 1: \mathcal{I}^{\prime} & <\mathcal{I}_{\text {opt }} \\
\operatorname{gain}\left(\mathcal{I}^{\prime}\right) & =\left(P_{\text {ret }}+P_{B}\right) S^{\prime}-\mathcal{I}^{\prime} \\
& =\left(P_{\text {ret }}+P_{B}\right) m^{+} \cdot \mathcal{I}^{\prime}-\mathcal{I}^{\prime} \\
& <\left(\left(P_{\text {ret }}+P_{B}\right) m^{+} \cdot \mathcal{I}^{\prime}-\mathcal{I}^{\prime}\right) \frac{\mathcal{I}_{\text {opt }}}{\mathcal{I}^{\prime}} \\
& =\left(P_{\text {ret }}+P_{B}\right) m^{+} \cdot \mathcal{I}_{\text {opt }}-\mathcal{I}_{\text {opt }} \\
& =\operatorname{gain}\left(\mathcal{I}_{\text {opt }}\right) .
\end{aligned}
$$

Case 2: $\mathcal{I}^{\prime}>\mathcal{I}_{\text {opt }}$. Since $\mathcal{I}^{\prime}>S_{\max } / m^{+}$, we have $S^{\prime}=S_{\max }$ (see Eq. 22). Then,

$$
\begin{aligned}
\operatorname{gain}\left(\mathcal{I}^{\prime}\right) & =\left(P_{\text {ret }}+P_{B}\right) S^{\prime}-\mathcal{I}^{\prime} \\
& =\left(P_{\text {ret }}+P_{B}\right) S_{\text {max }}-\mathcal{I}^{\prime} \\
& <\left(P_{\text {mkt }}+P_{B}\right) S_{\text {max }}-\mathcal{I}_{\text {opt }} \\
& =\left(P_{\text {mkt }}+P_{B}\right) m^{+} \cdot \mathcal{I}_{o p t}-\mathcal{I}_{o p t} \\
& =\operatorname{gain}\left(\mathcal{I}_{o p t}\right) .
\end{aligned}
$$

4) The upper bound of incentives: The highest incentives that a utility company can give to consumers while still experiences positive gain can be obtained by assuming the maximum increase in the consumption:

$$
\begin{aligned}
\left(P_{\text {ret }}+P_{B}\right) S_{\max }-\mathcal{I} & >0 \\
\left(P_{\text {ret }}+P_{B}\right) S_{\max } & >\mathcal{I},
\end{aligned}
$$

Example 3: Let us consider a case where the load demand $L=1000 k W h$ and the total energy generated in the abundance period $L^{+}=1500 \mathrm{kWh}$. In addition, let us assume that $P_{\text {ret }}=0.2 \$ / k W h, P_{B}=1 \$ / k W h$, and $m^{+}=1 k W h / \$$. Figure 3 shows company's gain for different amount of incentives provided to consumers. Note that, we have $S_{\max }=$ $1500 k W h-1000 k W h=500 k W h$. Maximum gain is obtained when the incentive is equal to $\mathcal{I}_{\text {opt }}=S_{\max } / \mathrm{m}^{+}=500 \$$. The highest incentives such that the company still experiences positive gain is $\left(P_{\text {ret }}+P_{B}\right) S_{\max }=600 \$$.

\section{RELATED WORK}

Borenstein and Holland analyzed the economics of realtime pricing (RTP) [10]. In particular, they assessed the market efficiency where both, RTP and flat-pricing scheme, coexist in the consumer base. They showed that increasing the number of consumers who adopt RTP scheme might harm the consumers who are already on RTP, but could bring advantage to consumers who stay in the flat-rate. There are also a number of studies that aim to foster the emergence of pervasive DR. However, most of them focused on price- 


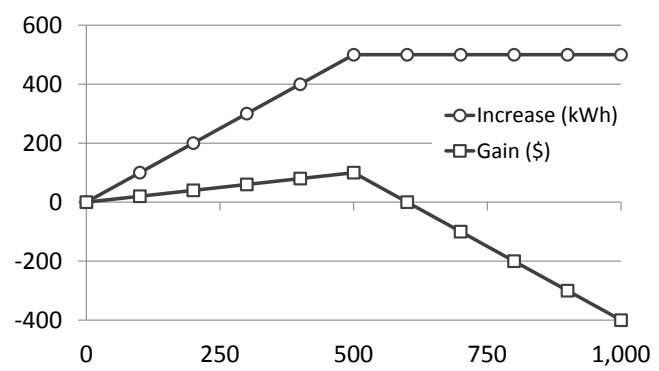

Fig. 3: Increase in energy consumption and gain for different incentives given to consumers in the case of DR to increase energy consumption, where $L=1000 \mathrm{kWh}, L^{+}=1500 \mathrm{kWh}, P_{\text {ret }}=$ $0.2 \$ / k W h, P_{B}=1 \$ / k W h$, and $m^{+}=1 k W h / \$$. The optimal incentive is $500 \$$, while the highest incentive for which the company still experiences positive gain is $600 \$$.

based mechanisms [7], [11]-[16]. These mechanisms require consumers to tirelessly track price fluctuation and adjust their consumption schedule accordingly to maximize their benefit (paying electricity bill as low as possible with the least inconvenience). To this end, some studies propose the use of software agents (or energy management systems) for automatic price monitoring and schedule optimization, and making the entire process seamless from the consumers' perspective [7], [13]-[16].

While this paper focuses on a more holistic view of incentive-based DR, there are also some studies dedicated to consumer baselines. In incentive-based DR, consumer baselines (or DR baselines) are estimates of what consumers would have consumed in the absence of DR events. It also plays an important role to determine the amount of incentives that consumers should receive [17]. Analyses of consumer baselines applied to large and commercial consumers have been discussed in [18]-[21], while its application to residential consumers has been discussed in [22].

\section{CONClusion ANd Future Work}

We have identified the conditions to benefit from pervasive DR, i.e., the lower bound of the consumer reduction/increase rate, $m$, the lower bound of the generation costs and market prices, the lower bound of the balancing prices, and the upper bound of the incentives. Furthermore, we have determined the optimal incentives to maximize gain. Although we model a single time period, the approach can be extended to multiple time periods by performing it iteratively.

It would certainly be interesting to find out how $m$ varies depending on various factors, or to accurately model the customer response function $r(\cdot)$. Additionally, to complicate matters, both could be noisy. Previous work has defined several customer models, but they are analytical models, are not based on real observations [22]. Accurately modeling both functions would require real project deployment, and it should be a large one so that the outcome is useful and generalizable. To start the project, however, utility companies would need the first insight on what to expect and how much incentives to provide. This paper aims to provide that first insight and a common ground/formalization to study the problem and enable one to craft better solutions.

\section{REFERENCES}

[1] M. H. Albadi and E. El-Saadany, "Demand response in electricity markets: An overview," in Power Engineering Society General Meeting, 2007. IEEE, June 2007, pp. 1-5.
[2] T. K. Wijaya, "Pervasive Data Analytics for Sustainable Energy Systems," Ph.D. dissertation, EPFL, Lausanne, 2015.

[3] Federal Energy Regulatory Commission, "Assessment of Demand Response \& Advanced Metering," Dec. 2010.

[4] B. Fogg, "A behavior model for persuasive design," in Proceedings of the 4th International Conference on Persuasive Technology, ser. Persuasive '09. New York, NY, USA: ACM, 2009, pp. 40:1-40:7.

[5] K. Kok, "The PowerMatcher: Smart Coordination for the Smart Electricity Grid," Ph.D. dissertation, Vrije Universiteit, Amsterdam, Germany, May 2013.

[6] A. Faruqui and S. George, "Quantifying Customer Response to Dynamic Pricing," The Electricity Journal, vol. 18, no. 4, pp. 53 - 63, 2005.

[7] A.-H. Mohsenian-Rad, V. Wong, J. Jatskevich, R. Schober, and A. Leon-Garcia, "Autonomous demand-side management based on game-theoretic energy consumption scheduling for the future smart grid," Smart Grid, IEEE Transactions on, vol. 1, no. 3, pp. 320-331, Dec 2010.

[8] M. Gonzalez Chapa and J. Vega Galaz, "An economic dispatch algorithm for cogeneration systems," in IEEE Power Engineering Society General Meeting, 2004, pp. 989-994 Vol.1.

[9] P. Rao and S. Nagendra, "Combined heat and power economic dispatch: A direct solution," Electric Power Components and Systems, vol. 34, no. 9, pp. 1043-1056, 2006.

[10] S. Borenstein and S. Holland, "On the Efficiency of Competitive Electricity Markets with Time-Invariant Retail Prices," RAND Journal of Economics, vol. 36, no. 3, pp. 469-493, Autumn 2005.

[11] Z. Chen, L. Wu, and Y. Fu, "Real-time price-based demand response management for residential appliances via stochastic optimization and robust optimization," Smart Grid, IEEE Transactions on, vol. 3, no. 4, pp. 1822-1831, Dec 2012.

[12] N. Li, L. Chen, and S. H. Low, "Optimal demand response based on utility maximization in power networks," in Power and Energy Society General Meeting, 2011 IEEE. IEEE, 2011, pp. 1-8.

[13] T. K. Wijaya, T. G. Papaioannou, X. Liu, and K. Aberer, "Effective consumption scheduling for demand-side management in the smart grid using non-uniform participation rate," in Sustainable Internet and ICT for Sustainability (SustainIT), 2013, Oct 2013, pp. 1-8.

[14] S. D. Ramchurn, P. Vytelingum, A. Rogers, and N. Jennings, "Agentbased control for decentralised demand side management in the smart grid," in The 10th International Conference on Autonomous Agents and Multiagent Systems - Volume 1, ser. AAMAS '11. Richland, SC: International Foundation for Autonomous Agents and Multiagent Systems, 2011, pp. 5-12.

[15] M. Vasirani and S. Ossowski, "Smart consumer load balancing: state of the art and an empirical evaluation in the spanish electricity market," Artificial Intelligence Review, vol. 39, no. 1, pp. 81-95, 2013.

[16] T. K. Wijaya, K. Larson, and K. Aberer, "Matching demand with supply in the smart grid using agent-based multiunit auction," in Communication Systems and Networks (COMSNETS), 2013 Fifth International Conference on, Jan 2013, pp. 1-6.

[17] S. Schnittger and S. Beare, "Economic implications of the proposed demand response mechanism," Australian Energy Market Commission, Tech. Rep., 2012.

[18] K. Coughlin, M. A. Piette, C. Goldman, and S. Kiliccote, "Statistical Analysis of Baseline Load Models for Non-Residential Buildings," Energy and Buildings, vol. 41, no. 4, pp. 374 - 381, 2009.

[19] “The Demand Response Baseline,” White Paper, EnerNOC, 2011.

[20] "PJM Empirical Analysis of Demand Response Baseline Methods," White Paper, KEMA, Inc., Apr. 2011.

[21] J. Mathieu, D. Callaway, and S. Kiliccote, "Examining uncertainty in demand response baseline models and variability in automated responses to dynamic pricing," in The 50th IEEE Conference on Decision and Control and European Control Conference (CDC-ECC), 2011, pp. 4332-4339.

[22] T. K. Wijaya, M. Vasirani, and K. Aberer, "When Bias Matters: An Economic Assessment of Demand Response Baselines for Residential Customers," IEEE Transactions on Smart Grid, 2014. 